Planetary Systems in the Universe - Observation, Formation and Evolution

Proceedings IAU Symposium No. 202, (c)2004 IAU

Alan Penny, Pawel Artymowicz, Anne-Marie Lagrange, \& Sara Russell, eds.

\title{
New extra-solar planets: the metallicity distribution revisited
}

\author{
Nuno C. Santos ${ }^{1}$, Garik Israelian ${ }^{2}$ and Michel Mayor ${ }^{1}$ \\ ${ }^{1}$ Observatoire de Genève, CH1290 Sauverny, Switzerland \\ ${ }^{2}$ Instituto de Astrofisica de Canarias, 38200 La Laguna, Tenerife, Spain
}

\begin{abstract}
We present the spectroscopy results of a new set of planetary host stars discovered in the context of the Geneva extra-solar planet search programme. The analysis was based on high $\mathrm{S} / \mathrm{N}$ spectra obtained with the CORALIE spectrograph at the 1.2-m Euler Swiss Telescope (at La Silla, ESO). We revisit the metallicity distribution of stars with planets. The present sample, largely enriched by the inclusion of the most recent discoveries, strongly suggests that stars with planets are anomalously metal rich, but that the source of the metallicity excess is most probably "primordial".
\end{abstract}

\section{Spectroscopy}

In a previous work (Santos, Israelian, \& Mayor 2000) we have presented the spectroscopic analysis of 8 stars known to harbor low mass companions. Here we continue the study, and we present our abundance results for 5 other objects, namely HD 19994, HD 108147, HD 121504, HD 162020, and HD 168746.
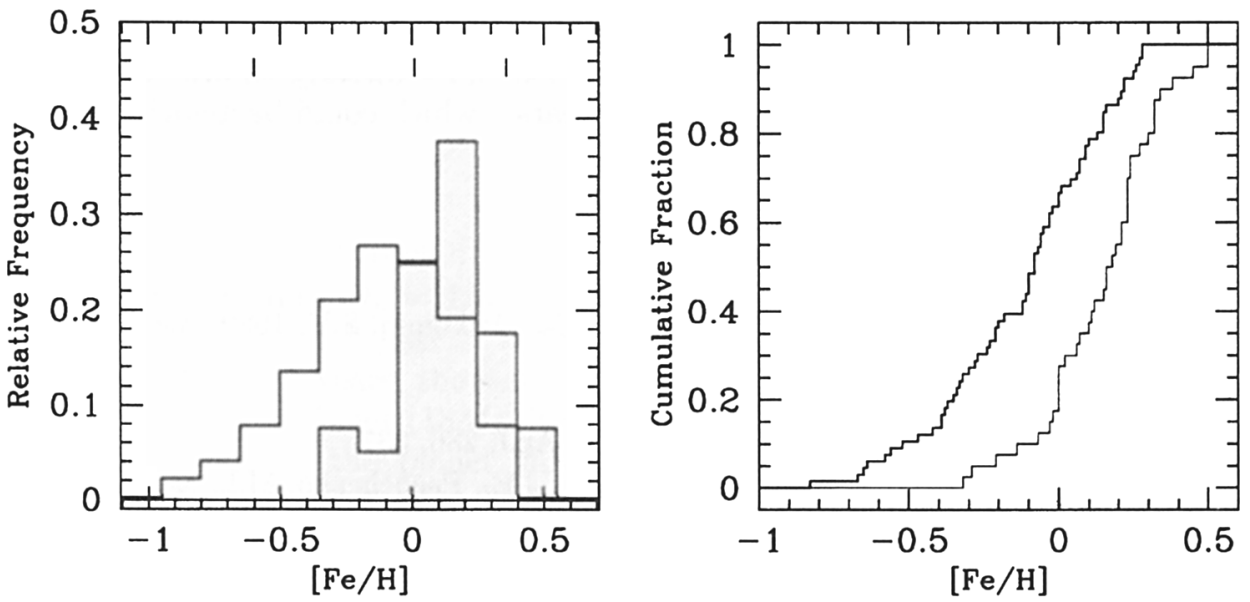

Figure 1. Distribution of stars with planets (shaded histogram) compared with the distribution of field $G$ and early $K$ dwarfs (Favata et al. 1997). Vertical lines represent stars with brown-dwarf companions. A KolmogorovSmirnov test (right panel) shows that the probability that the two samples belong to the same population is lower than $10^{-5}$. 
Table 1. Derived abundances and atmospheric parameters.

\begin{tabular}{llccc}
\hline \multicolumn{1}{c}{ Star } & $T_{\text {eff }}(\mathrm{K})$ & $\log g\left(\mathrm{~cm} \mathrm{~s}^{-2}\right)$ & $\xi_{t}\left(\mathrm{~km} \mathrm{~s}^{-1}\right)$ & {$[\mathrm{Fe} / \mathrm{H}]$} \\
\hline HD 1237 & $5540 \pm 75$ & $4.70 \pm 0.20$ & $1.47 \pm 0.1$ & $+0.10 \pm 0.08$ \\
HD 13445 & $5180 \pm 75$ & $4.75 \pm 0.25$ & $0.79 \pm 0.1$ & $-0.21 \pm 0.07$ \\
HD 19994 & $6160 \pm 50$ & $4.25 \pm 0.25$ & $1.49 \pm 0.1$ & $+0.23 \pm 0.06$ \\
HD 52265 & $6060 \pm 50$ & $4.29 \pm 0.25$ & $1.29 \pm 0.1$ & $+0.21 \pm 0.06$ \\
HD 75289 & $6140 \pm 50$ & $4.51 \pm 0.20$ & $1.47 \pm 0.1$ & $+0.28 \pm 0.07$ \\
HD 82943 & $6010 \pm 50$ & $4.62 \pm 0.20$ & $1.08 \pm 0.1$ & $+0.32 \pm 0.06$ \\
HD 83443 & $5460 \pm 100$ & $4.55 \pm 0.25$ & $1.05 \pm 0.1$ & $+0.38 \pm 0.11$ \\
HD 108147 & $6260 \pm 50$ & $4.65 \pm 0.20$ & $1.39 \pm 0.1$ & $+0.19 \pm 0.07$ \\
HD 121504 & $6080 \pm 50$ & $4.79 \pm 0.20$ & $1.32 \pm 0.1$ & $+0.16 \pm 0.07$ \\
HD 162020 & $4830 \pm 100$ & $4.76 \pm 0.20$ & $0.72 \pm 0.1$ & $+0.01 \pm 0.11$ \\
HD 168746 & $5590 \pm 50$ & $4.55 \pm 0.20$ & $0.97 \pm 0.1$ & $-0.07 \pm 0.08$ \\
HD 169830 & $6300 \pm 50$ & $4.11 \pm 0.25$ & $1.37 \pm 0.1$ & $+0.21 \pm 0.05$ \\
HD 202206 & $5750 \pm 75$ & $4.80 \pm 0.20$ & $0.96 \pm 0.1$ & $+0.36 \pm 0.08$ \\
\hline
\end{tabular}

For other elements refer to Santos, Israelian, \& Mayor (2000), and the poster version of this proceedings at http://obswww.unige.ch/ santos/iau_feh_poster.ps.gz

We used a standard LTE atmosphere analysis with a revised version of the line abundance code MOOG (Sneden 1973), and a grid of Kurucz et al. (1993) ATLAS9 atmospheres. The results are summarized in Table 1. We took $\log \epsilon_{\odot}=5.47$ for Fe.

\section{The metallicity distribution}

In Fig. 1 we present an histogram of the metallicity distribution of stars with planets when compared with the same distribution of field $\mathrm{G}$ and $\mathrm{K}$ dwarfs (Favata, Micela, \& Sciortino 1997). The addition of the objects listed in the Table shows that the trend persists: stars with planets really seem to be more metal-rich than field dwarfs (Gonzalez 1998). We are comparing, however, two samples whose $[\mathrm{Fe} / \mathrm{H}]$ were determined using distinct techniques; an uniform study (currently under way) may be important to confirm the observed trends, but we do not think that the differences will disappear.

We have also studied the abundances of $\mathrm{C}, \mathrm{O}$ and $\alpha$-elements $\mathrm{Ti}, \mathrm{Ca}$, and $\mathrm{Si}$, for the stars listed in the Table. The analysis did not reveal any significant differences between stars with planets and field stars. In all cases, a more refined and uniform analysis with better precision $(<0.1 \mathrm{dex})$ is necessary to unveil eventual differences in the two populations.

\section{Primordial abundance vs enrichment}

Since the mass of the convective envelope of a solar-type dwarf increases as we go from $\mathrm{F}$ to $\mathrm{K}$ dwarfs, if the enrichment scenario is the key of the observed chemical anomalies, we might expect an anti-correlation of the $[\mathrm{Fe} / \mathrm{H}]$ values with the masses of the convective envelopes at the time of planetary formation. 


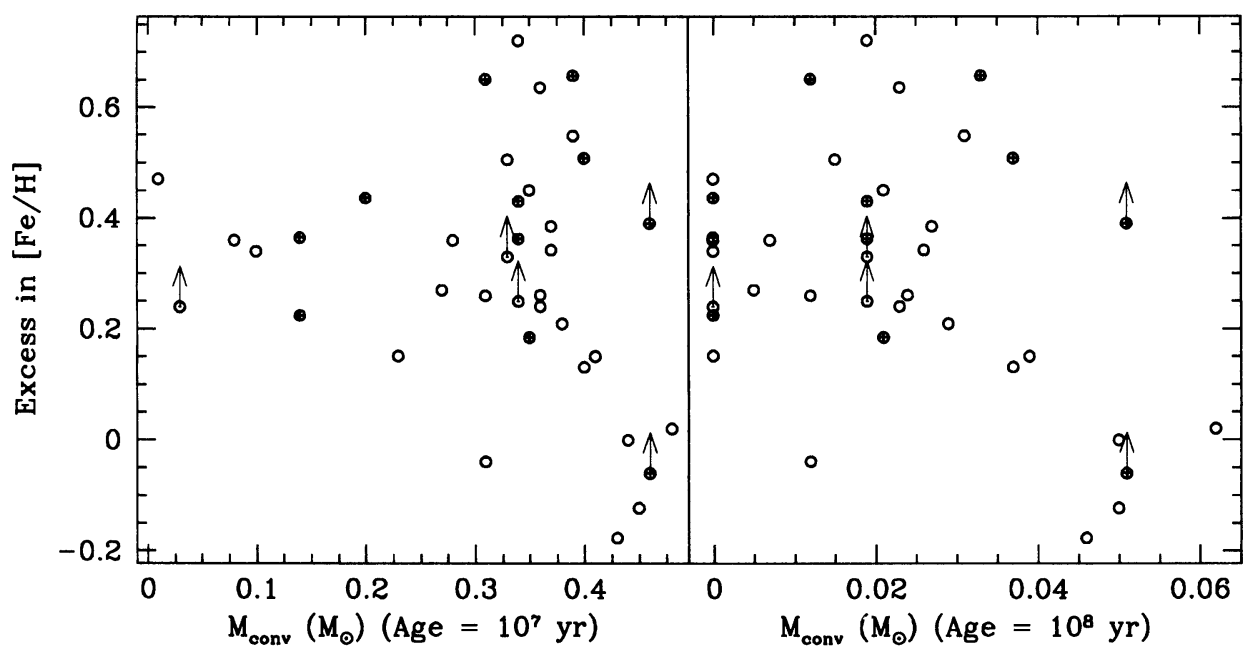

Figure 2. Plot of the $[\mathrm{Fe} / \mathrm{H}]$ as a function of the mass of the convective envelope for two different stellar ages. "Earth" symbols denote stars with planets orbiting closer than $\sim 0.08 \mathrm{AU}$. Arrows represent objects for which no age was estimated, and no metallicity age gradient correction was applied.

In Fig. 2 we plot the value of the $[\mathrm{Fe} / \mathrm{H}]$ excess for the stars hosting extrasolar planets against the mass of the convective envelope at the time of planet formation $\left(\sim 10^{7} \mathrm{yr}\right.$, Zuckerman et al. 1995) and at $10^{8} \mathrm{yr}$. The masses for the convective envelope were taken from D'Antona \& Mazzitelli (1994). Here, $[\mathrm{Fe} / \mathrm{H}]$ excess denotes the value of $[\mathrm{Fe} / \mathrm{H}]$ corrected for the galactic age gradient.

No significant trends are observed in the plots. These results, together with the high metallicity excesses observed, do not support the enrichment scenario alone (at least a simple one), neither during planetary formation (e.g. by disk accretion) nor after this period (e.g. by the fall of one or more planets into the star due to dynamical instability in a multiple system). However, this does not exclude the contribution of such a process in some particular cases.

\section{References}

D'Antona, F., \& Mazzitelli, I. 1994, ApJS, 90, 467

Favata, F., Micela, G., \& Sciortino, S. 1997, A\&A, 323, 809

Gonzalez, G. 1998, A\&A, 334, 221

Kurucz, R.L., Furenlid, I., Brault, J., \& Testerman, L. 1984, Solar Flux Atlas from 296 to $1300 \mathrm{~nm}$, NOAO Atlas No. 1

Santos, N.C., Israelian, G., \& Mayor, M. 2000, A\&A, in press

Sneden, C., 1973, Ph.D. thesis, University of Texas

Zuckerman, B., Forveille, T., \& Kastner, J.H. 1995, Nature, 373, 494 